\title{
Ranking Investment Projects ${ }^{1}$
}

\author{
James E. Foster \\ Department of Economics, Vanderbilt University, Nashville, TN 37235 \\ james.e.foster@vanderbilt.edu
}

Fax: 615.343.2391

and

\section{Tapan Mitra}

Department of Economics, Cornell University, Ithaca, NY 14853

tm19@cornell.edu

Fax: 607.255.2818

April, 2001

\footnotetext{
${ }^{1}$ We thank Robert Driskill, Andrea Maneschi, Roy Radner, and participants of seminars at NYU, Notre Dame, Purdue, and Washington University for helpful comments. Foster is grateful for support from the John D. and Catherine T. MacArthur Foundation through its network on Inequality and Poverty in Broader Perspective.
} 


\begin{abstract}
This paper describes conditions under which one investment project dominates a second project in terms of net present value, irrespective of the choice of the discount rate. The resulting partial ordering of projects has certain similarities to stochastic dominance. However, the structure of the net present value function leads to characterizations that are quite specific to this context. Our theorems use Bernstein's (1915) innovative results on the representation and approximation of polynomials, as well as other general results from the theory of equations, to characterize the partial ordering. We also show how the ranking is altered when the range of discount rates is limited or the rate varies period by period. Journal of Economic Literature Classification Numbers: D92, G31, H043, O22.

Key Words: investment projects; present value; stochastic dominance; polynomials; rate of return over cost; time dominance.
\end{abstract}




\section{INTRODUCTION}

Most public or private investment projects entail a significant up-front investment with a stream of returns over many subsequent periods. The traditional way of reckoning the economic viability of such a project is to use a discount rate to convert future earnings into present values, which are then weighed against initial cost to obtain the discounted present value of the project. The choice of discount rate is crucial. If it is low enough, future earnings will essentially receive full weight, which entails a positive present value for the typical project. At higher discount rates, future earnings receive less importance, and the present value falls into the negative range. Clearly, the acceptability of a given project can be sensitive to the specific rate chosen.

The discount rate is no less critical when comparing two competing (mutually exclusive) investment projects. If one project $\boldsymbol{a}$ entails both a smaller initial investment and lower returns down the road than another project $\boldsymbol{b}$, then a higher discount rate will tend to favor project $\boldsymbol{a}$ due to its smaller initial investment, while a lower rate will favor $\boldsymbol{b}$ 's higher returns over time. In other words, the difference in present values of the two projects is the same as the present value of the stream of differences in returns; and since this stream is initially negative (as $\boldsymbol{b}$ has higher initial investment) and subsequently positive (as $\boldsymbol{b}$ has higher returns), the present value of the differences is positive or negative depending on the discount rate. The relative ranking of two projects can be sensitive to the chosen rate. ${ }^{2}$

Now, how easy is it to select an appropriate discount rate? For private investment projects, this selection is in theory straightforward, since it can be based on an observable market rate of interest facing the firm or individual. ${ }^{3}$ On the other hand, public projects are evaluated

\footnotetext{
2 This point was emphasized by Fisher (1930) and later by Alchian (1955) in his critique of Keynes' marginal efficiency of capital.

${ }^{3}$ See Hirshleifer (1958, p.336), however, for a discussion of whether it is the borrowing rate or the lending rate (or something in between) that ought to be used. He also provides references, in the capital budgeting literature, where either the borrowing rate or the lending rate has been favored as the correct rate to use.
} 
using a social rate of discount, which is considerably more difficult to ascertain. Indeed, there is an extensive and varied literature addressing the question of finding the right social discount rate. ${ }^{4}$ And while there may be general agreement that the social discount rate is not just a market rate, there is substantially less agreement on exactly how it should be determined. It should come as no surprise that, in the absence of a generally accepted method for determining the social discount rate, a wide range of rates can often be observed in actual use. ${ }^{5}$ This calls into question the robustness of comparisons that involve only a single discount rate.

The present paper asks: When does one project unambiguously dominate another in the sense that it has a higher present value at all discount rates? To be sure, any projects $\boldsymbol{a}$ and $\boldsymbol{b}$ for which $\boldsymbol{a}$ has uniformly lower costs and higher returns than $\boldsymbol{b}$ could be so ranked. ${ }^{6}$ But, in fact, the possibilities for unambiguous ranking go well beyond simple period-by-period dominance. Consider a comparison of Project $\boldsymbol{a}$, costing $\$ 10$ million and yielding $\$ 5$ million after one year and $\$ 8$ million after two, and Project $\boldsymbol{b}$, costing $\$ 12$ million with respective returns of $\$ 10$ million and $\$ 3$ million. At a discount rate of, say, 5\% the present values of $\boldsymbol{a}$ and $\boldsymbol{b}$ are about $\$ 2$ million and $\$ 250,000$, respectively, and so $\boldsymbol{a}$ is better than $\boldsymbol{b}$ given this rate. Moreover, it can be shown that the relative ranking of $\boldsymbol{a}$ and $\boldsymbol{b}$ is preserved no matter which discount rate is used. For this comparison, and indeed many others, the choice of discount rate is not an issue.

The goal of this paper is to describe the conditions under which one investment project dominates another independent of the choice of the discount rate. Implicitly we are characterizing a binary ranking of projects that is clearly transitive (if $\boldsymbol{a}$ dominates $\boldsymbol{a}^{\prime}$ while $\boldsymbol{a}^{\prime}$ dominates $\boldsymbol{a}^{\prime \prime}$, then $\boldsymbol{a}$ dominates $\boldsymbol{a}^{\prime \prime}$ ) and irreflexive (no project dominates itself), but is not complete, since certain pairs cannot be so ranked. The proper term for such a transitive, irreflexive, but not necessarily complete ranking is partial ordering. There are certain

\footnotetext{
4 See, for example, Lind (1982) and the papers cited therein.

${ }^{5}$ Lind (1982, p.4-6), for example, provides a relevant quote from a U.S. Government document that complains of discount rates effectively ranging from 0 to infinity. ${ }^{6}$ Mishan (1971, p. 185), for example, considers situations of this sort, and uses the terminology "dominates" to describe the relationship between them. Fisher (1930, p. 151) uses the term "ineligible" to describe an option that is "out of the question, whatever be the rate of interest."
} 
similarities to the other well-known partial orderings in economics, including the Lorenz ranking (see for example Sen, 1997), the variable-line poverty orderings of Foster and Shorrocks (1988a,b), and the stochastic dominance rankings from expected utility analysis (see Bawa (1975)). But the techniques used here are, for the most part, different. ${ }^{7}$

We begin in Section 2 with the various definitions and notation used in setting up the problem. Dominance of one project over another is restated as requiring the polynomial whose coefficients are the differences in cash flows of the two projects to be positive on the (open) unit interval. Section 3 offers several partial characterizations of the dominance ranking. In particular, the Bernstein form $^{8}$ of a polynomial is introduced, made up of "Bernstein coefficients," derived from the original polynomial's coefficients, and transformations of the variable that are positive on the relevant domain. A sufficient condition for unambiguous dominance is obtained in terms of the signs of the Bernstein coefficients. We show that this condition is both necessary and sufficient in the special case where all roots of the original polynomial are known to be real.

Section 4 obtains complete characterizations for "short duration" projects. We employ discriminant conditions to determine when all roots of the resulting polynomial are real (and thus the above characterization can be applied) and when some roots are not real (in which case "brute force" may be used). We take advantage of the low dimension to offer graphical representations of the set of comparable projects to give a rough indication of the completeness of the related partial ordering.

The next section presents a complete characterization based closely on the work of Bernstein (1915). The idea is that any $n^{\text {th }}$ degree polynomial can be interpreted as an $m^{\text {th }}$ degree polynomial (for $m>n$ ) with the last $m-n$ coefficients being zero. The $m^{\text {th }}$ degree polynomial has a Bernstein form representation, which is also equivalent to the original $n^{\text {th }}$ order polynomial. Hence there is an infinite number of Bernstein representations of a given

\footnotetext{
7 We note below (in Sections 3 and 6) an interesting relationship between two of our conditions and the first two degrees of stochastic dominance.

${ }^{8}$ See, for example, Cargo and Shisha (1966).
} 
polynomial, each with its own set of $m+1$ coefficients that yield another sufficient condition for unambiguous dominance. By a theorem in Bernstein (1915), this approach also produces a general necessary condition: if the unambiguous dominance condition holds, then there exists an $m \geq n$, for which the $m+1$ Bernstein coefficients have the required signs. In sum, then, $\boldsymbol{a}$ dominates $\boldsymbol{b}$ exactly in the case that some Bernstein form has nonnegative coefficients (with at least one coefficient being strictly positive).

Our unambiguous criterion is in a sense too strong since it requires dominance at all nonnegative discount rates, including some that may be outside of anyone's relevant range. In Section 6 we consider an alternative dominance condition that only requires dominance over a restricted range. Since unanimity is now required for a smaller set of rates, the resulting partial ordering can make more comparisons or, equivalently, is "more complete". We derive the corresponding limited-range conditions and illustrate the extent of their additional coverage. Section 7 offers a second extension of our methodology to the case of discount rates, which vary over time. Interestingly, we find that depending on the types of time paths of discount rates that are allowed, the two strongest sufficient conditions obtained in the fixed discount rate case are actually necessary and sufficient for the variable discount rate case.

Our results are quite closely related to several strands of literature, including work by Pratt and Hammond (1979) on the existence and number of "internal rates of return", papers by Bøhren and Hansen (1980) and Ekern (1981) on a more general concept of "time dominance", and certain results from mathematics (e.g., Hausdorff (1921) and Pratt (1979)) on roots of polynomials. Section 8 offers a discussion of this related literature. The final section concludes with some suggestions for further work.

\section{THE SETTING}

The two projects to be compared will be represented by their return (or cash flow) vectors $\boldsymbol{a}=\left(a_{0}, a_{1}, \ldots, a_{n}\right) \in \mathbb{R}^{n+1}$ and $\boldsymbol{b}=\left(b_{0}, b_{1}, \ldots, b_{n}\right) \in \mathbb{R}^{n+1}$, which indicate the magnitude of the return for each period from 0 to $n$, and whether the return is positive (a net 
income) or negative (a net outflow). For simplicity of notation, all projects are represented as having the same finite horizon $n$; if the projects have different horizons, the shorter vector can be augmented with zero entries to reconcile the size difference. We will often use vector dominance to rank vectors, where vector $\boldsymbol{a}$ dominates vector $\boldsymbol{b}$ if $a_{k} \geq b_{k}$ for all $k=0,1, \ldots, n$, with $a_{k}>b_{k}$ for some $k$. In this case we write $\boldsymbol{a}>\boldsymbol{b}$.

Given a rate of discount $r>0$, one can compare the two projects in terms of present value by dividing the return in period $k$ by $(1+r)^{k}$ and summing over all periods. We will find it convenient to work with the corresponding discount factor $x=1 /(1+r)$, where $0<x<1$, so that $x^{k}$ is the coefficient on period $k$ 's return in the present value expression. We use $P_{\boldsymbol{a}}(x)$ to denote the present value of a given project $\boldsymbol{a}$ at discount factor $x$, so that $P_{\boldsymbol{a}}(x)=a_{0}+a_{1} x+\ldots+a_{n} x^{n}$. This converts the present value expression into a polynomial in the variable $x$, and allows certain results from the theory of equations to be applied.

We are interested in finding necessary and sufficient conditions so that the present value of $\boldsymbol{a}$ is larger than the present value of $\boldsymbol{b}$ for all discount rates $r>0$, or equivalently, for all discount factors $0<x<1$. In symbols, the problem is to find conditions on the projects $\boldsymbol{a}=$ $\left(a_{0}, a_{1}, \ldots, a_{n}\right)$ and $\boldsymbol{b}=\left(b_{0}, b_{1}, \ldots, b_{n}\right)$ such that

$$
P_{\boldsymbol{a}}(x)>P_{\boldsymbol{b}}(x) \quad \text { for all } 0<x<1
$$

or alternatively

$$
\sum_{k=0}^{n} a_{k} x^{k}>\sum_{k=0}^{n} b_{k} x^{k} \quad \text { for all } 0<x<1
$$

Define the net project $c$ by

$$
\boldsymbol{c}=\left(c_{0}, c_{1}, \ldots, c_{n}\right)=\boldsymbol{a}-\boldsymbol{b}=\left(a_{0}-b_{0}, a_{1}-b_{1}, \ldots, a_{n}-b_{n}\right)
$$

The problem can be reformulated as determining conditions on the net project $\boldsymbol{c}$ for which

$$
P_{c}(x):=\sum_{k=0}^{n} c_{k} x^{k}>0 \quad \text { for all } 0<x<1
$$


In other words, we are interested in conditions under which the present value of $c$ is always positive. This equivalent formulation will be particularly helpful in the proofs of the various characterizations, and for evaluating the completeness of the implied ranking of projects.

\section{SOME PARTIAL CHARACTERIZATIONS}

It is quite clear that two necessary conditions for $P_{\boldsymbol{a}}(x)>P_{\boldsymbol{b}}(x)$ to hold independent of $x$ are

$$
a_{0} \geq b_{0}
$$

(by letting $x \rightarrow 0$ in (1)), and

$$
\sum_{k=0}^{n} a_{k} \geq \sum_{k=0}^{n} b_{k}
$$

(by letting $x \rightarrow 1$ in (1)). In other words, the initial return and the mean (or total) return must be as high in project $\boldsymbol{a}$ as in project $\boldsymbol{b}$. These conditions represent the two extremes for $x$; the first where $x$ approaches 0 and the future is totally discounted, and the second where $x$ approaches 1 and the future is given the same weight as today. One might expect the actual $x$ to be bounded away from one or the other of these limits. If so, these conditions would no longer be necessary; see Section 6 below.

An obvious sufficient condition under which dominance condition (1) will hold independent of $x$ is

$$
\begin{array}{ll}
a_{k} \geq b_{k} \quad & \text { for all } k=0,1, \ldots, n \\
& \text { with strict inequality for some } k .
\end{array}
$$

What this requires is that $\boldsymbol{a}$ have a higher return than $\boldsymbol{b}$ in some period, and no lower return in every period. Of course, this period-by-period dominance is unlikely to be useful for most applications. To develop a more promising sufficient condition, we proceed as follows. 
For $k=0,1, \ldots, n$, let $A_{k}$ denote the partial sum $\left(a_{0}+\ldots+a_{k}\right)$, and similarly let $B_{k}:=$ $\left(b_{0}+\ldots+b_{k}\right)$. Then by Abel's partial summation formula (see Rudin $(1976, \mathrm{p} .70)$ ), we obtain

$$
\sum_{k=0}^{n} a_{k} x^{k}=A_{0}(1-x)+A_{1}\left(x-x^{2}\right)+\ldots .+A_{n-1}\left(x^{n-1}-x^{n}\right)+A_{n} x^{n}
$$

for $\boldsymbol{a}$ and an analogous expression for $\boldsymbol{b}$. Thus, a sufficient condition under which $P_{\boldsymbol{a}}(x)>P_{\boldsymbol{b}}(x)$ will hold independent of $x$ is

$$
\begin{array}{ll}
A_{k} \geq B_{k} & \text { for all } k=0,1, \ldots, n \\
& \text { with strict inequality for some } k .
\end{array}
$$

In other words, the sufficient condition is that for some $k$, the partial sum of the first $k$ returns is higher in $\boldsymbol{a}$ than in $\boldsymbol{b}$, and for no $k$ is it higher in $\boldsymbol{b}$. Note that (3b) contains the two necessary conditions given above since the first partial sum condition is $a_{0} \geq b_{0}$ while the final partial sum condition is $a_{0}+\ldots+a_{n} \geq b_{0}+\ldots+b_{n}$. Moreover, (3b) is implied by the period-by-period sufficient condition (3a), but has much greater scope for application. As an example, consider the projects $\boldsymbol{a}=(-2,2,3,4,3)$ and $\boldsymbol{b}=(-6,3,4,6,2)$. We see from condition (3b) that unambiguous dominance holds -- a conclusion that is hard to guess from an examination of the vectors $\boldsymbol{a}$ and $\boldsymbol{b}$ themselves. One can therefore recognize (3b) as a potentially powerful sufficient condition.

We note that these conditions are rather closely related to the well-known criteria of first and second order stochastic dominance used in the analysis of risk. ${ }^{9}$ Indeed, if $\boldsymbol{a}$ and $\boldsymbol{b}$ were ordered vectors (from lowest to highest returns) with the interpretation that each entry is an equi-probable payoff, then (3a) would indicate that $\boldsymbol{a}$ dominates $\boldsymbol{b}$ according to first order stochastic dominance, while ( $3 b$ ) would ensure that $\boldsymbol{a}$ dominates $\boldsymbol{b}$ according to second order stochastic dominance. Similarly, (2) would represent the twin necessary conditions for first or

\footnotetext{
9 This fact has been noted elsewhere in the literature. See Section 8.
} 
second order stochastic dominance: that the lowest payoff and (respectively) the mean payoff must be no lower in $\boldsymbol{a}$ than in $\boldsymbol{b}$.

One way to depict second order stochastic dominance is with the help of Shorrocks' (1983) generalized Lorenz curve, which plots $(1 / n)$ times the $k^{\text {th }}$ partial sum against $k / n$ and connects the points. When the vectors are ordered, this curve represents second order stochastic dominance in that a higher curve is associated with a dominating vector. When the vectors are not ordered, the curve is no longer a generalized Lorenz curve, but what might instead be called a generalized concentration curve. ${ }^{10}$ Clearly the generalized concentration curve provides a representation of sufficient condition (3b) in that a higher curve implies dominance according to our unambiguous criterion. The curves associated with projects $\boldsymbol{a}=(-2,2,3,4,3)$ and $\boldsymbol{b}=(-6$, 3, 4, 6, 2), for example, are given in Figure 1, with project $\boldsymbol{a}$ clearly dominating project $\boldsymbol{b}$. Note also that the generalized concentration curve also illustrates the necessary conditions (2) since the curve's slope at 0 is the initial period's return and the intercept at 1 is the mean return. This relationship with stochastic dominance will be examined further in Section 7.

\section{[FIG. 1. Generalized concentration curves.]}

Consider once again the net project $\boldsymbol{c}=\boldsymbol{a}-\boldsymbol{b}$ with its associated net returns $\left(c_{0}, \ldots, c_{n}\right)$ and partial sums $\left(C_{0}, \ldots, C_{n}\right)$. The necessary conditions (2) may be restated as $C_{0} \geq 0$ and $C_{n} \geq 0$, while the sufficient conditions ( $3 \mathrm{~b})$ are $C_{k} \geq 0$ for $k=0, \ldots, n$. There is clearly a good deal of room between these two sets of conditions. We now show that the sufficient conditions can be sharpened substantially, while the necessary conditions can likewise be strengthened in a particular case.

Toward this end, let us define an alternative representation for polynomials that is particularly well-suited for the problem at hand. Let $s=\left(s_{0}, \ldots, s_{n}\right)$ be any vector in $\mathbb{R}^{n+1}$ and consider the polynomial function

\footnotetext{
${ }^{10}$ For a discussion of the concentration curve and the generalized Lorenz curve, see Lambert (1993).
} 


$$
\begin{aligned}
B_{s}(x) & =s_{0}(1-x)^{n}+s_{1} x(1-x)^{n-1}+\ldots .+s_{n-1} x^{n-1}(1-x)+s_{n} x^{n} \\
& =\sum_{k=0}^{n} s_{k} x^{k}(1-x)^{n-k}
\end{aligned}
$$

for $x \in \mathbb{R} . B_{\boldsymbol{S}}(x)$ is called a Bernstein polynomial or a Bernstein form, following Bernstein (1915) who showed that every polynomial $P_{\boldsymbol{c}}(x)$ can be expressed as $B_{\boldsymbol{S}}(x)$ for some $\boldsymbol{s}$ in $\mathbb{R}^{n+1}$.

This proposition is straightforward for $n=1$ since $c_{0}+c_{1} x=s_{0}(1-x)+s_{1} x$, where $s_{0}=c_{0}$ and $s_{1}=c_{0}+c_{1}$. Similarly for $n=2$ we have

$$
\begin{aligned}
c_{0}+c_{1} x+c_{2} x^{2} & =\left[c_{0}(1-x)+\left(c_{0}+c_{1}\right) x\right]+c_{2} x^{2} \\
& =\left[c_{0}(1-x)+\left(c_{0}+c_{1}\right) x\right](1-x)+\left[c_{0}(1-x)+\left(c_{0}+c_{1}\right) x\right] x+c_{2} x^{2} \\
& =s_{0}(1-x)^{2}+s_{1} x(1-x)+s_{2} x^{2},
\end{aligned}
$$

where $s_{0}=c_{0}, s_{1}=2 c_{0}+c_{1}$, and $s_{3}=c_{0}+c_{1}+c_{2}$. Bernstein (1915) used a recursive argument on $n$ to show that in general

$$
\begin{aligned}
P_{\boldsymbol{c}}(x) & =s_{0}(1-x)^{n}+s_{1} x(1-x)^{n-1}+\ldots+s_{n-1} x^{n-1}(1-x)+s_{n} x^{n} \\
& =B_{\boldsymbol{S}}(x)
\end{aligned}
$$

where $s=\left(s_{0}, \ldots, s_{n}\right)$ is defined by

$$
s_{k}=\left(\begin{array}{c}
n \\
n-k
\end{array}\right) c_{0}+\left(\begin{array}{c}
n-1 \\
n-k
\end{array}\right) c_{1}+\ldots+\left(\begin{array}{c}
n-k \\
n-k
\end{array}\right) c_{k} \quad \text { for } k=0,1, \ldots, n .
$$

The Bernstein coefficients $s_{k}$ are thus weighted sums of returns from net project $c$, where the weights are found along the diagonal of Pascal's triangle. In other words, $s=c \Pi$, where $\Pi$ is the $(n+1) \times(n+1)$ matrix

$$
\Pi=\left[\begin{array}{ccccc}
\left(\begin{array}{c}
n \\
n
\end{array}\right) & \left(\begin{array}{c}
n \\
n-1
\end{array}\right) & \ldots & \left(\begin{array}{c}
n \\
1
\end{array}\right) & \left(\begin{array}{c}
n \\
0
\end{array}\right) \\
0 & \left(\begin{array}{c}
n-1 \\
n-1
\end{array}\right) & \ldots & \left(\begin{array}{c}
n-1 \\
1
\end{array}\right) & \left(\begin{array}{c}
n-1 \\
0
\end{array}\right) \\
\vdots & \vdots & & \vdots & \vdots \\
0 & 0 & \ldots & \left(\begin{array}{c}
1 \\
1
\end{array}\right) & \left(\begin{array}{c}
1 \\
0
\end{array}\right) \\
0 & 0 & \ldots & 0 & \left(\begin{array}{l}
0 \\
0
\end{array}\right)
\end{array}\right]
$$

whose nonzero entries form an inverted Pascal's triangle. For example, if $\boldsymbol{c}=$ $(2,-5,5,-2)$, then 


$$
s=(2,-5,5,-2)\left[\begin{array}{llll}
1 & 3 & 3 & 1 \\
0 & 1 & 2 & 1 \\
0 & 0 & 1 & 1 \\
0 & 0 & 0 & 1
\end{array}\right]=(2,1,1,0)
$$

In addition, since the matrix $\Pi$ is nonsingular, it follows immediately that the Bernstein representation is unique; for any given $c$, there is only one vector $s$ such that $P_{c}(x)=B_{s}(x)$, and that vector is the vector of Bernstein coefficients. ${ }^{11}$ We can summarize these observations in the following result.

LEMMA 1. (Bernstein) Let $\boldsymbol{c}, \boldsymbol{s} \in \mathbb{R}^{n+1}$. Then $P_{\boldsymbol{c}}(x)=B_{\boldsymbol{s}}(x)$ if and only if $\boldsymbol{s}=\mathrm{c} \Pi$.

Notice that in the above example, $s=(2,1,1,0)$ is a nonnegative vector with all entries positive but one. The corresponding Bernstein polynomial $B_{S}(x)=2(1-x)^{3}+(1-x)^{2} x+(1-x) x^{2}+$ $0 x^{n}$ is positive for $0<x<1$, which then ensures that for the original net project $c=(2,-5,5,-2)$ we have $P_{c}(x)>0$ for $0<x<1$, our unambiguous dominance condition (1). In general suppose that for net project $c$, the vector $s$ of Bernstein coefficients is nonnegative with at least one positive entry; i.e., $s=c \Pi>0$. Since the terms $(1-x)^{n-k}$ and $x^{k}$ in $B_{\boldsymbol{s}}(x)=\Sigma_{k} s_{k} x^{k}(1-x)^{n-k}$ are always strictly positive for $0<x<1$, it follows that $P_{c}(x)$ is positive for $0<x<1$, and we thus obtain:

THEOREM 1. Suppose that net project $\boldsymbol{c}=\boldsymbol{a}-\boldsymbol{b}$ satisfies $\boldsymbol{c} \Pi>0$. Then project $\boldsymbol{a}$ unambiguously dominates project $\boldsymbol{b}$.

Theorem 1 yields a remarkably simple sufficient condition for unambiguous dominance of $\boldsymbol{a}$ over $\boldsymbol{b}$ : check whether $\boldsymbol{c} \Pi>0$ or, equivalently, whether $\boldsymbol{a} \Pi$ dominates $\boldsymbol{b} \Pi$ according to the

\footnotetext{
${ }^{11}$ Suppose that there is a vector $s^{\prime}$ in $\mathbb{R}^{n+1}$ such that $P_{\boldsymbol{c}}(x)=B_{\boldsymbol{S}^{\prime}}(x)$ for all $x$. Since $\Pi$ is nonsingular, we can obtain a project $c^{\prime}=s^{\prime} \Pi-1$ which has $\boldsymbol{s}^{\prime}$ as its vector of Bernstein coefficients, and hence $P_{\boldsymbol{c}^{\prime}}(x)=B_{\boldsymbol{S}}(x)=P_{\boldsymbol{c}}(x)$ for all $x \in(0,1)$, which holds only if $\boldsymbol{c}=\boldsymbol{c}^{\prime}$. Consequently $s=s^{\prime}$.
} 
vector dominance relation. Moreover, this condition has far greater coverage than the (unweighted) partial sum condition (3b). To see this, note that (3b) can be written as $c \Gamma>0$, where

$$
\Gamma=\left[\begin{array}{ccccc}
1 & 1 & \ldots & 1 & 1 \\
0 & 1 & \ldots & 1 & 1 \\
\therefore & . . & \ldots & . . & . . \\
0 & 0 & \ldots & 1 & 1 \\
0 & 0 & \ldots & 0 & 1
\end{array}\right]
$$

In contrast to $\Gamma$, each column of $\Pi$ is strictly decreasing in its nonzero entries and, consequently, gives even more weight to returns in earlier periods. For example, when $n=4$, the requirements " $\boldsymbol{\Gamma}>0$ " and "c $\Pi>0$ " become, respectively,

\begin{tabular}{|c|c|c|}
\hline$c_{0}$ & $\geq 0$ & $c_{0}$ \\
\hline$c_{0}+c_{1}$ & $\geq 0$ & $4 c_{0}+c_{1}$ \\
\hline$c_{0}+c_{1}+c_{2}$ & $\geq 0$ and & $6 c_{0}+3 c_{1}+c_{2}$ \\
\hline$c_{0}+c_{1}+c_{2}+c_{3}$ & $\geq 0$ & $4 c_{0}+3 c_{1}+2 c_{2}+c_{3}$ \\
\hline$c_{0}+c_{1}+c_{2}+c_{3}+c_{4}$ & $\geq 0$ & $c_{0}+c_{1}+c_{2}+c_{3}+c_{4}$ \\
\hline
\end{tabular}

(where at least one of each set of inequalities is strict). It is clear that every inequality on the right side can be verified by applying a combination of inequalities from the left, so that "c $\Gamma>$ 0 " implies " $c \Pi>0$ "; the converse, however, is not true.

Despite this improved applicability, the condition given in Theorem 1 is still not necessary in general. For example, as we shall see below, the net project $c=(2,-5,5)$ satisfies condition (1); however, $s=c \Pi=(2,-1,2)$, which clearly violates the requirement " $c \Pi>0$ " of Theorem 1. For a reasonably large class of net projects, though, we can show that "c $>0$ " is indeed necessary. The key assumption concerns the roots of the polynomial $P_{\boldsymbol{c}}(x)$.

THEOREM 2. Suppose that the net project $\boldsymbol{c}=\boldsymbol{a}-\boldsymbol{b}$ is such that the polynomial $P_{\boldsymbol{c}}(x)=\sum_{k=0}^{n} c_{k} x^{k}$ has only real roots. Then project $\boldsymbol{a}$ unambiguously dominates project $\boldsymbol{b}$ if and only if $\boldsymbol{c} \Pi>0$. 
Proof. By Theorem 1, we need only prove necessity. So suppose that $P_{c}(x)$ has only real roots and that (1) holds. We are to show that the corresponding vector $s=\left(s_{0}, \ldots, s_{n}\right)=c \Pi$ of Bernstein coefficients satisfies $s>0$.

Consider the polynomial

$$
Q(z)=s_{n}+s_{n-1} z+s_{n-2} z^{2}+\ldots+s_{1} z^{n-1}+s_{0} z^{n},
$$

where the variable $z$ is explicitly taken to be complex. Using the Binomial Theorem for complex numbers (see, for example, Knopp (1952, p. 25)), we obtain

$$
Q(z)=c_{0}(1+z)^{n}+c_{1}(1+z)^{n-1}+\ldots+c_{n-1}(1+z)+c_{n} .
$$

Now if $z \neq-1$, then defining $x=1 /(1+z)$ we obtain

$$
Q(z)=\left(c_{0} / x^{n}\right)+\left(c_{1} / x^{n-1}\right)+\ldots+\left(c_{n-1} / x\right)+c_{n},
$$

and hence

$$
Q(z) x^{n}=P_{c}(x)
$$

where $P_{c}(x)=\Sigma_{k} c_{k} x^{k}$ is now explicitly taken to be a polynomial of a complex variable $x$. Given any arbitrary (possibly complex) root $z_{0} \neq-1$ of $Q(z)$, it follows that $x_{0}=1 /\left(1+z_{0}\right)$ is a root of $P_{c}(x)$. And since all roots of $P_{c}(x)$ are real, this implies that $z_{0}$ must also be real. Consequently, all roots of $Q(z)$ are real. Note further that if $Q(z)$ were to have a positive root $z_{0}$, then $x_{0}=$ $1 /\left(1+z_{0}\right)$ would satisfy $P_{c}\left(x_{0}\right)=0$ and $0<x_{0}<1$, contrary to condition (1). Therefore, $Q(z)$ can have no positive real roots, which by an application of Descartes' Rule of Signs (e.g., Dickson (1939, p. 77 and problem 15 on p. 80)) implies that the coefficients $s_{k}$ must have no variations of sign, or equivalently, that all nonzero coefficients $s_{k}$ of $Q(z)$ must have the same sign. Moreover, since $P_{\boldsymbol{c}}(1 / 2)>0$, it follows that $Q(1)=s_{n}+s_{n-1}+s_{n-2}+\ldots+s_{1}+s_{0}>0$, which yields $\boldsymbol{s}>0$, as desired.

Thus, in the very special case where the polynomial $P_{c}(x)$ only has real roots, the sufficient conditions become necessary as well. Of course, the practical import of Theorem 2 is compromised by the fact that a determination of the roots of $P_{c}(x)$ is actually very close to the original problem itself. For once we find all roots, we can check directly whether they all are 
outside of the interval $(0,1)$, in which case the sign of $P_{c}(x)$ will be constant on $(0,1)$, and $P_{c}(x)$ will have the same sign as, say, $P_{c}(1 / 2)$, for all $x \in(0,1)$. So we turn to a slightly different approach to finding complete characterizations of our unambiguous ranking.

\section{COMPLETE CHARACTERIZATIONS FOR $n \leq 3$}

We now examine the special cases where $n=1,2$, and 3, each of which leads to a tractable set of necessary and sufficient conditions. These characterizations also reveal the structure of the ranking and, in particular, provide an initial look into its completeness or ability to make comparisons. We begin with the simplest case of $n=1$ where the relevant polynomial is $P_{c}(x)=c_{0}+c_{1} x$. In this case, the sufficient partial sum condition ( $\left.3 \mathrm{~b}\right)$ becomes $c_{0} \geq 0$ and $c_{0}$ $+c_{1} \geq 0$ (with one of the inequalities being strict). Moreover, necessary conditions (2) may be expressed as $c_{0} \geq 0$ and $c_{0}+c_{1} \geq 0$, while it is clear that strict dominance requires one of these inequalities to be strict (otherwise $c$ would be the zero vector). Consequently, (3b) constitutes a set of necessary and sufficient conditions for dominance to hold in the case $n=1$.

[FIG. 2. Dominance for $n=1$.]

Figure 2 depicts the collection of net projects $c=\left(c_{0}, c_{1}\right)$ satisfying these conditions. Note that the darker gray area is the set of all net projects satisfying sufficient condition (3a), namely $c_{0} \geq 0$ and $c_{1} \geq 0$ (with at least one strict inequality), while the light gray area indicates the additional net projects captured by (3b).

The analysis of the case $n=2$ is somewhat more complicated. We state the necessary and sufficient conditions in the form of a theorem.

THEOREM 3. Suppose that net project $\boldsymbol{c}=\boldsymbol{a}-\boldsymbol{b}$ is of length $\boldsymbol{n}=2$ and satisfies $c_{2} \neq$ 0 . Then project $\boldsymbol{a}$ unambiguously dominates project $\boldsymbol{b}$ if and only if either $(i)$ condition $\boldsymbol{c} \Pi>$ 0 holds or else (ii) $4 c_{0} c_{2}-c_{1}^{2}>0$ and necessary conditions (2) jointly hold. 
Proof. When $n=2$, the net project is $c=\left(c_{0}, c_{1}, c_{2}\right)$, and $P_{c}(x)=c_{0}+c_{1} x+c_{2} x^{2}$ becomes the relevant polynomial. Suppose that condition (1) holds. Assuming that $4 c_{0} c_{2}-c_{1}^{2} \leq 0$, we know from the quadratic formula that all roots of $P_{\boldsymbol{c}}(x)$ are real. Then by Theorem 2, it follows that $c \Pi>0$. Alternatively, if we assume that $4 c_{0} c_{2}-c_{1}^{2}>0$, then since conditions (2) are necessary for dominance, we are done with necessity.

Now suppose that (i) or (ii) holds. If $c \Pi>0$, then by Theorem 2, dominance condition (1) must follow. Alternatively, if conditions (2) and $4 c_{0} c_{2}-c_{1}^{2}>0$ together hold, then by the quadratic formula, all roots of $P_{c}(x)$ are complex. Consequently $P_{c}(0)=c_{0}$ must be strictly positive, as must be $P_{c}(x)$ for all $0<x<1$ by continuity of $P_{c}(x)$, and thus condition (1) holds.

[FIG. 3. Dominance for $n=2$.]

It is easy to depict the set of projects leading to dominance for the case $n=2$ and this is done in Figure 3 for arbitrary $c_{0}$. The set of $\left(c_{1}, c_{2}\right)$ satisfying (2) and $4 c_{0} c_{2}-c_{1}^{2}>0$ is given by the lightly shaded area above the parabola. The set of additional pairs satisfying $c \Pi>0$ is found in the shaded area below and to the right of the parabola. The shaded region in Figure 3 is thus the set of projects leading to dominance. For example, the project $(2,-5,5)$ clearly lies within this region as can be seen by plotting $\left(c_{1}, c_{2}\right)=(-5,5)$ in Figure 3 , where $c_{0}=2$. In the special case where $c_{0}=0$, the condition $4 c_{0} c_{2}-c_{1}^{2}>0$ cannot hold and we are left with a $\left(c_{1}\right.$, $c_{2}$ )-space version of Figure 2.

The last short horizon projects we will consider have length $n=3$, with relevant polynomial $P_{c}(x)=c_{0}+c_{1} x+c_{2} x^{2}+c_{3} x^{3}$; for simplicity we assume $c_{3} \neq 0$. Let

$$
\delta=\left[c_{3}{ }^{2} c_{0}-\left(c_{1} c_{2} c_{3} / 3\right)+2\left(c_{2} / 3\right)^{3}\right]^{2}-4\left[\left(c_{2} / 3\right)^{2}-\left(c_{1} c_{3} / 3\right)\right]^{3} .
$$

It can be shown (see, for example, Uspensky (1948, p. 87)) that $\delta$ is the appropriate cubic discriminant term, with $\delta>0$ indicating that $P_{c}(x)$ has two complex roots and one real root, while $\delta \bullet 0$ ensures that all the roots of $P_{c}(x)$ are real. We have the following result. 
THEOREM 4. Suppose that net project $\boldsymbol{c}=\boldsymbol{a}-\boldsymbol{b}$ is of length $n=3$ and satisfies $c_{3} \neq$ 0 . Then project $\boldsymbol{a}$ unambiguously dominates project $\boldsymbol{b}$ if and only if either $(i)$ condition $\boldsymbol{c} \Pi$ $>0$ holds or else (ii) $\delta>0$ and necessary conditions (2) jointly hold.

Proof. Let $n=3$ and suppose that dominance condition (1) holds. If $\delta \leq 0$, then since the roots of $P_{c}(x)$ are real, we know by Theorem 2 that $c \Pi>0$ must hold. Alternatively, if we assume that $\delta>0$, then since (2) is necessary for dominance, we are done with the proof of necessity.

Now suppose that (i) or (ii) holds. If $\boldsymbol{c} \Pi>0$, then by Theorem 1 dominance condition (1) must follow. Alternatively, suppose that $\delta>0$ and conditions (2) hold. We know from $\delta>0$ that two roots of $P_{c}(x)$ are complex and one is real. Since $c_{3} \neq 0$, we either have $c_{3}>0$ or $c_{3}<$ 0 . If $c_{3}>0$, then $P_{c}(x)<0$ for sufficiently negative $x$. Moreover since $P_{c}(0)=c_{0} \geq 0$, we know by continuity that the real root of $P_{c}(x)$ must be non-positive. Consequently $P_{c}(1)=c_{0}+c_{1}+c_{2}$ $+c_{3}$ is strictly positive, as must be $P_{c}(x)$ for all $0<x<1$ by continuity of $P_{c}(x)$, and thus condition (1) holds. If $c_{3}<0$, we have $P_{c}(x)<0$ when $x$ is sufficiently positive. Moreover since $P_{c}(1)=c_{0}+c_{1}+c_{2}+c_{3} \geq 0$, we know by continuity that the real root of $P_{c}(\mathrm{x})$ lies in the interval $[1, \infty)$. Consequently $P_{\boldsymbol{c}}(1)=c_{0}+c_{1}+c_{2}+c_{3}$ must be strictly positive, as must be $P_{c}(x)$ for all $0<x<1$ by continuity of $P_{c}(x)$, and thus condition (1) holds.

The approach used in this section has led to clear-cut conditions for dominance in the special case where $n \leq 3$. However, the discriminant condition for $n=3$ is cumbersome, and any similar attempts for $n>3$ are unlikely to yield tractable conditions. In the next section we follow a different approach, which, rather than identifying a self-contained set of necessary and sufficient conditions, yields a series of ever-tightening sufficient conditions which in the limit become necessary.

\section{A GENERAL CHARACTERIZATION}


Consider the net project $c=(2,-5,5)$ with horizon $n=2$. The sufficient condition of Theorem 1 clearly fails, since

$$
\boldsymbol{c}\left[\begin{array}{lll}
1 & 2 & 1 \\
0 & 1 & 1 \\
0 & 0 & 1
\end{array}\right]=(2,-1,2)
$$

and yet a quick check of the necessary and sufficient conditions from Theorem 3 confirms that $c$ satisfies the dominance condition. Clearly $c \Pi>0$ misses this case. However, suppose that we express $c$ as the equivalent project $c^{3}:=(2,-5,5,0)$ with horizon 3 , and apply the sufficiency test of Theorem 1 for this "longer horizon" project. It is easily confirmed that

$$
s^{3}:=c^{3}\left[\begin{array}{llll}
1 & 3 & 3 & 1 \\
0 & 1 & 2 & 1 \\
0 & 0 & 1 & 1 \\
0 & 0 & 0 & 1
\end{array}\right]=(2,1,1,2)
$$

and so $P_{c^{3}}(x)>0$ for $0<x<1$, and since $P_{c}(x)$ is identical to $P_{c^{3}}(x)$, this verifies that $c$ satisfies condition (1).

In general, for any net project $c$ of length $n$, one can construct an equivalent project $c^{m}$ of length $m \geq n$ by appending $m-n$ zeroes to $c$, and thus obtaining an alternative Bernstein representation of $P_{\boldsymbol{c}}(x)$ and a new vector of coefficients $\boldsymbol{s}^{m}=\left(s_{0}^{m}, s_{1}^{m}, \ldots, s_{m}^{m}\right)$ to use with Theorem 1. Actually, there is a somewhat more direct way of constructing $s^{m}$ from $c$. Let $\Pi_{m}$ be the $(n+1) \times(m+1)$ truncated Pascal matrix formed from the $(m+1) \times(m+1)$ Pascal matrix by removing the last $m-n$ rows. Since the final $m-n$ entries in the vector $c^{m}$ are 0 , we know that $s^{m}=c \Pi_{m}$. In the above example, for instance,

$$
\Pi_{3}=\left[\begin{array}{llll}
1 & 3 & 3 & 1 \\
0 & 1 & 2 & 1 \\
0 & 0 & 1 & 1
\end{array}\right],
$$

and clearly $s^{3}=(2,1,1,2)=(2,-5,5) \Pi_{3}=c \Pi_{3}$. Given these observations, then, we would expect "c $\Pi_{m}>0$ for some $m \geq n$ " to be a sufficient condition for unambiguous dominance.

What is not so obvious is that that this is a necessary condition as well. To see this we will need an additional lemma, also due to Bernstein (1915). 
LEMMA 2. (Bernstein) For any given project $c \in \mathbb{R}^{n+1}$ and every $m \geq n$, define $\varepsilon^{m}:=\max _{k}\left|\left[s_{k}^{m} /\left(\begin{array}{c}m \\ m-k\end{array}\right)\right]-P_{\boldsymbol{c}}\left(\frac{k}{m}\right)\right|$. Then $\lim _{m \rightarrow \infty} \varepsilon^{m}=0$.

The proof is given in the appendix.

Lemma 2 shows that the Bernstein coefficients, $s_{k}^{m}$, are quite closely linked to the $m+1$ values $P_{\boldsymbol{c}}\left(\frac{k}{m}\right)$, for $0 \leq k \leq m$. Indeed, for large enough $m$, the true value $P_{\boldsymbol{c}}\left(\frac{k}{m}\right)$ is approximated by $\left[s_{k}^{m} /\left(\begin{array}{c}m \\ m-k\end{array}\right)\right]$. Consequently, whenever $P_{\boldsymbol{c}}\left(\frac{k}{m}\right)$ is positive and the approximation is good enough, the associated Bernstein coefficient, $s_{k}^{m}$, must be positive as well. This is the basis for the following complete characterization.

THEOREM 5. Suppose that net project $\boldsymbol{c}=\boldsymbol{a}-\boldsymbol{b}$ is of arbitrary length $n$. Then project $\boldsymbol{a}$ unambiguously dominates project $\boldsymbol{b}$ if and only if $\boldsymbol{c} \Pi_{m}>0$ for some $m \geq n$.

Proof. Suppose that $c \Pi_{m}>0$ for some $m \geq n$. Then we know that $s^{m}=c \Pi_{m}$ is the associated vector of Bernstein coefficients for $\boldsymbol{c}^{m}$, where $\boldsymbol{c}^{m}$ is the $m$-horizon project found by appending $m-n$ zeroes to $\boldsymbol{c}$. Consequently, from Theorem 1, condition (1) holds for $\boldsymbol{c}^{m}$ and since $P_{\boldsymbol{c}}(x)=P_{\boldsymbol{c}^{m}}(x)$ for all $x$, it holds for $\boldsymbol{c}$ as well.

Conversely, suppose that dominance condition (1) holds for $c$, so that $P_{c}(x)>0$ for $0<x$ <1. $P_{\boldsymbol{c}}(x)$ can be expressed as $P_{\boldsymbol{c}}(x)=f(x) g(x)$ where $f(x)=x^{i}(1-x)^{j}$ (for $i, j \geq 0$ ) is a polynomial whose only roots (if any) are 0 and 1, while $g(x)$ is a polynomial for which 0 and 1 are not roots. In addition, $g(x)$ can be written as $g(x)=\bar{c}_{0}+\cdots+\bar{c}_{N} x^{N}$, where $N=n-i-j$ and $\bar{c}=\left(\bar{c}_{0}, \ldots, \bar{c}_{N}\right)$ $\in \mathbb{R}^{N+1}$, and consequently we may denote $P_{\bar{c}}(x)=g(x)$. Clearly $P_{\bar{c}}(x)>0$ for $0<x<1$. By continuity, $P_{\bar{c}}(0) \geq 0$ and $P_{\bar{c}}(1) \geq 0$, and since 0 and 1 are not roots for $P_{\bar{c}}(x)$, we must have $P_{\bar{c}}(x)>0$ for $0 \leq x \leq 1$.

The minimum value of the continuous function $P_{\bar{c}}(x)$ on the unit interval must be strictly positive, and hence we can find $\varepsilon>0$ such that $P_{\bar{c}}(x)>\varepsilon$ for $0 \leq x \leq 1$. By Lemma 2, and the definition of convergence, there exists $M \geq N$ such that the Bernstein coefficients $\bar{c} \Pi_{M}$ $=\overline{\boldsymbol{s}}^{M}=\left(\bar{s}_{0}^{M}, \ldots, \bar{s}_{M}^{M}\right)$ satisfy $\left|\left[\bar{s}_{k}^{M} /\left(\begin{array}{l}M \\ M-k\end{array}\right)\right]-P_{\bar{c}}(k / M)\right|<\varepsilon / 2$ for $k=0, \ldots, M$. Thus 
$\left[\bar{s}_{k}^{M} /\left(\begin{array}{l}M \\ M-k\end{array}\right)\right]>0$, and since $\left(\begin{array}{l}M \\ M-k\end{array}\right)>0$ for every $k$, we obtain $\bar{s}_{k}^{M}>0$. Hence $\overline{\boldsymbol{s}}^{M}=$ $\left(\bar{s}_{0}^{M}, \ldots, \bar{s}_{M}^{M}\right)$ is a strictly positive vector. Moreover, by Lemma 1 we know that $P_{\bar{c}^{M}}(x)=$ $B_{\overline{\boldsymbol{s}} \boldsymbol{M}}(x)$ and so

$$
P_{\bar{c}}(x)=\sum_{k=0}^{M} \bar{s}_{k}^{M} x^{k}(1-x)^{M-k}
$$

Consequently,

$$
P_{c}(x)=x^{i}(1-x)^{j} P_{\bar{c}}(x)=\sum_{k=0}^{M} \bar{s}_{k}^{M} x^{i+k}(1-x)^{j+M-k}=\sum_{k=0}^{m} t_{k}^{m} x^{k}(1-x)^{m-k}
$$

where $m:=M+i+j$ and $\boldsymbol{t}^{m}=\left(t_{0}^{m}, t_{1}^{m}, \ldots, t_{m}^{m}\right)$ is defined by

$$
t_{k}^{m}=\left\{\begin{array}{cc}
\bar{s}_{k-i}^{M} & \text { for } k=i, \ldots, i+M \\
0 & \text { otherwise }
\end{array}\right.
$$

But then $P_{\boldsymbol{c}^{m}}(x)=B_{\boldsymbol{t}^{m}}(x)$, which by Lemma 1 implies that $\boldsymbol{t}^{m}=\boldsymbol{s}^{m}$, the vector of Bernstein coefficients associated with $c^{m}$. Thus, $c \Pi_{m}=\boldsymbol{s}^{m}=\boldsymbol{t}^{m}>0$, as desired.

Theorem 5 shows how the partial characterization provided in Theorem 1 can be extended to a complete characterization. Theorem 1 was based on an $n+1$ dimensional vector $s$ of Bernstein coefficients that ensures dominance condition (1) whenever $s>0$. From Theorem 5 we see that $s=s^{n}$ is but one of a collection $\left\{s^{m} \in \mathbb{R}^{m+1} \mid m \geq n\right\}$ of vectors that may be used to indicate dominance; and whenever dominance holds, we must have $s^{m}>0$ for all $m$ large enough. In addition, each vector $\boldsymbol{s}^{m}$ is easily constructed from the net project $c$ with the help of the truncated Pascal matrix $\Pi_{m}$ and this, in turn, allows us to restate the result in terms of the original projects $\boldsymbol{a}$ and $\boldsymbol{b}$ as follows: $\boldsymbol{a}$ dominates $\boldsymbol{b}$ if and only if there exists $m \geq n$ for which $\boldsymbol{a} \Pi_{m}>\boldsymbol{b} \Pi_{m}$. So, for example, $\boldsymbol{a}=(-10,1,9,4)$ dominates $\boldsymbol{b}=(-12,8,1,5)$, since $\boldsymbol{a} \Pi_{5}=(-5,-24,-$ $37,-13,14,9)>\boldsymbol{b} \Pi_{4}=(-7,-27,-37,-14,10,7)$.

We should also mention that there is a simple recursive method of deriving the vector $\boldsymbol{s}^{m+1}$ from $\boldsymbol{s}^{m}$ for $m \geq n$. First, it is clear that $\boldsymbol{s}_{0}^{m}=\boldsymbol{s}_{0}^{m+1}=c_{0}$ and $\boldsymbol{s}_{m}^{m}=\boldsymbol{s}_{m+1}^{m+1}=c_{0}+\ldots+c_{n}$. Second, the recursive property of Pascal's triangle ensures that $s_{j}^{m+1}=s_{j-1}^{m}+s_{j}^{m}$ for $j=1, \ldots$, 
$m$. Thus $s^{m+1}$ is found from $s^{m}$ using an adjacent sum algorithm that is analogous to the way the Pascal triangle is constructed. For example, if we begin with $c=(2,-7,8,-1)$, we obtain $s=s^{3}=$ $(2,-1,0,2)$. Then $s^{4}=(2,1,-1,2,2), s^{5}=(2,3,0,1,4,2)$, and $s^{6}=(2,5,3,1,5,6,2)$, and so forth. A clear implication is that once $s^{m}>0$ for some $m \geq n$, we know that $s^{m^{\prime}}>0$ for all $m^{\prime}>m$.

FIG. 4. Successive approximation and dominance.

The above recursive definition leads to an interesting geometrical interpretation of the procedure underlying Lemma 2 and Theorem 5. As noted above, the value $\left[\boldsymbol{s}_{k}^{m} /\left(\begin{array}{c}m \\ m-k\end{array}\right)\right]$ is a proxy for the true value of the function $P_{\boldsymbol{c}}(x)$ at $x=(k / m)$. For any given $m \geq n$, consider the piecewise linear function $f^{m}(x)$ found by plotting the $m+1$ points $\left(k / m,\left[s_{k}^{m} /\left(\begin{array}{c}m-k \\ m-1\end{array}\right)\right.\right.$ and connecting adjacent pairs with linear segments. It is clear from Lemma 2 that as $m$ goes to infinity, $f^{m}(x)$ tends to the original polynomial $P_{\boldsymbol{c}}(x)$ at each $x \in[0,1]$. However, we can provide an even more concrete description of the process by which these piecewise linear functions come about. It is clear that $f^{m+1}(x)=f^{m}(x)=P_{c}(x)$ at $x=0$ and $x=1$; all approximating functions take on the same value -- the true functional value -- at each of the two limiting points. It turns out that $f^{m+1}(x)=f^{m}(x)$ at all points of the form $x=k /(m+1)$, where $k=0, \ldots, m+1$. To see this, note that for $k=1, \ldots, m$ we have

$$
\begin{aligned}
f^{m+1}\left(\frac{k}{m+1}\right) & =\left[s_{k}^{m+1} /\left(\begin{array}{c}
m+1 \\
m-k+1
\end{array}\right)\right]=\left[s_{k-1}^{m} /\left(\begin{array}{c}
m+1 \\
m-k+1
\end{array}\right)\right]+\left[s_{k}^{m} /\left(\begin{array}{c}
m+1 \\
m-1
\end{array}\right)\right] \\
& =\frac{k}{m+1}\left[s_{k-1}^{m} /\left(_{m-k+1}^{m}\right)\right]+\frac{m+1-k}{m+1}\left[s_{k}^{m} /\left(_{m-k}^{m}\right)\right] \\
& =f^{m}\left(\frac{k}{m+1}\right) .
\end{aligned}
$$

Consequently, each $f^{m+1}$ (with $m>n$ ) can be constructed from the previous approximating function $f^{m}$ by an intuitive algorithm: Plot the $m+2$ points $\left(k /(m+1), f^{m}(k /(m+1))\right)$ for $k=0, \ldots$, $m$ and connect the adjacent pairs with line segments. Figure 4 shows how this process evolves for the project $c=(2,-7,8,-1)$. Although the true function is positive on the interval $(0,1)$, the initial approximation functions dip into negative values. Eventually, though, the approximating functions draw near enough to $P_{c}(x)$ and the defining values, $s_{k}^{m}$, become nonnegative (with 
some positive), as required by Theorem 5. The precise value of $m$ where this takes place is not specified by the theorem; it could be relatively small, as in this example, or large. We will return to this question in Section 9.

\section{LIMITED RANGE RESULTS}

The motivation for this study is that there is often disagreement on the precise discount rate to be used in evaluating investment projects; in such a situation, it is useful to develop criteria under which a project is unambiguously better at all rates of discount. But even when complete agreement on the precise rate is impossible, there may be a reasonable range over which there is general agreement. For example, while one may argue whether $r=5 \%$ or $r=8 \%$ is appropriate, there may be strong agreement that $r$ should not fall short of $1 \%$ nor exceed $25 \%$. This leads to a change in our dominance condition, and a concomitant reexamination of our characterization results thus far.

Suppose that the limited range of discount rates translates into a range $(u, v)$ in discount factor space, where $0<u<v<1$. For example, the restriction that $r$ falls between $1 \%$ and $25 \%$ translates (approximately) to the requirement that $x \in(u, v)=(0.80,0.99)$. With such a restricted range we can expect to obtain conditions in terms of $c, u$, and $v$ that have much greater applicability than the unrestricted conditions derived above. In fact, we can show that by a simple affine transformation of variables, this new problem can be converted into the form of the original problem, and that greater applicability arises when the unrestricted range solution is converted back.

Consider the transformation $y:=\frac{x-u}{v-u}$ from $x \in(u, v)$ to $y \in(0,1)$. Substituting $u+$ $(v-u) y$ for $x$ in $P_{c}(x)$ yields

$$
\begin{aligned}
& P_{c}(x)=c_{0}+c_{1}(u+(v-u) y)+\ldots+ \\
=c_{0}\left(\begin{array}{l}
0 \\
0
\end{array}\right)+c_{1}\left(\left(\begin{array}{c}
1 \\
0
\end{array}\right) u+\left(\begin{array}{c}
1 \\
1
\end{array}\right)(v-u) y\right)+\ldots & +c_{k}\left[\left(\begin{array}{c}
k \\
0
\end{array}\right) u^{k}+\left(\begin{array}{c}
k \\
1
\end{array}\right) u^{k-1}(v-u) y+\ldots+\left(\begin{array}{c}
k \\
k
\end{array}\right)(v-u)^{k} y^{k}\right]+\ldots \\
& +c_{n}\left[\left(\begin{array}{c}
n \\
0
\end{array}\right) u^{n}+\left(\begin{array}{c}
n \\
1
\end{array}\right) u^{n-1}(v-u) y+\ldots+\left(\begin{array}{c}
n \\
n
\end{array}\right)(v-u)^{n} y^{n}\right]
\end{aligned}
$$




$$
\begin{aligned}
& =\left[c_{0}\left(\begin{array}{l}
0 \\
0
\end{array}\right)+c_{1}\left(\begin{array}{l}
1 \\
0
\end{array}\right) u+c_{2}\left(\begin{array}{l}
2 \\
0
\end{array}\right) u^{2}+\ldots+c_{n}\left(\begin{array}{c}
n \\
0
\end{array}\right) u^{n}\right]+\left[c_{1}\left(\begin{array}{l}
1 \\
1
\end{array}\right)+c_{2}\left(\begin{array}{l}
2 \\
1
\end{array}\right) u+\ldots+c_{n}\left(\begin{array}{c}
n \\
1
\end{array}\right) u^{n-1}\right](v-u) y+\ldots \\
& \quad+\left[c_{k}\left(\begin{array}{c}
k \\
k
\end{array}\right)+c_{k+1}\left(\begin{array}{c}
k+1 \\
k
\end{array}\right) u+\ldots+c_{n}\left(\begin{array}{c}
n \\
k
\end{array}\right) u^{n-k}\right](v-u)^{k} y^{k}+\ldots+c_{n}\left(\begin{array}{c}
n \\
n
\end{array}\right)(v-u)^{n} y^{n} \\
& =q_{0}+q_{1} y+\ldots+q_{n} y^{n},
\end{aligned}
$$

where

$$
q_{k}=\left[c_{k}\left(\begin{array}{c}
k \\
k
\end{array}\right)+c_{k+1}\left(\begin{array}{c}
k+1 \\
k
\end{array}\right) u+\ldots+c_{n}\left(\begin{array}{c}
n \\
k
\end{array}\right) u^{n-k}\right](v-u)^{k} \quad \text { for } k=0,1, \ldots, n
$$

Viewing $\boldsymbol{q}=\left(q_{0}, q_{1}, \ldots, q_{n}\right)$ as a net project in its own right, we can express the unambiguous dominance condition for $c$ on the restricted range $(u, v)$ in terms of the usual dominance condition for $\boldsymbol{q}$ on $(0,1)$. This formal relationship between the two is summarized in the next theorem.

THEOREM 6. Let $\boldsymbol{c}=\boldsymbol{a}-\boldsymbol{b}$ be any net project and let $\boldsymbol{q}$ be the project defined in (4).

Then

$$
P_{c}(x)>0 \text { for all } x \in(u, v)
$$

is equivalent to

$$
P_{\boldsymbol{q}}(y)>0 \text { for all } y \in(0,1) \text {. }
$$

Consequently, any of the previous results characterizing dominance condition (1) can be applied to the derived project $\boldsymbol{q}$ in order to capture the limited range dominance condition for $\boldsymbol{c}$.

To see the extent of additional coverage for limited range results, consider projects $\boldsymbol{a}$ and $\boldsymbol{b}$ defined by $\boldsymbol{a}=(-6,8)$, and $\boldsymbol{b}=(-2,3)$, so that the net project $\boldsymbol{c}=\boldsymbol{a}-\boldsymbol{b}=(-4,5)$. Then, it is easy to see that $P_{c}(x)<0$ for $x \in(0,0.8)$ and $P_{c}(x)>0$ for $x \in(0.8,1)$. Thus, neither project is unambiguously better than the other at all discount factors $x \in(0,1)$. However, suppose, to follow up on the discussion given above, there is agreement that the discount rate certainly falls between $1 \%$ and $25 \%$, so that the discount factor falls between 0.80 and 0.99 . Then, clearly, project $\boldsymbol{a}$ dominates project $\boldsymbol{b}$, for the agreed upon range of discount factors $(0.80,0.99)$. It can 
be easily checked for this example that $\boldsymbol{q}=(0,0.95)$, so that $P_{\boldsymbol{q}}(y)>0$ for all $y \in(0,1)$, in accordance with Theorem 6, where $y=[(x-0.8) / 0.19]$ for $x \in(0.8,0.99)$.

\section{VARIABLE RATES OF DISCOUNT}

A key assumption of the above analysis is that the discount rate, though unknown, is fixed throughout the life of the investment. We now consider a generalization to the case where the rate may vary from period to period. There are two main ways of characterizing a variable rate environment. The first approach allows the per-period discount factor to vary over time, and uses the product of the per-period factors to discount future returns. This approach ensures that greater weight is given to cash flows received in earlier periods. The second directly assigns every future period a separate discount factor within the unit interval, which is then used to evaluate that period's returns. This admits additional possibilities, including a preference for future returns over current returns. We now turn to the characterizations of the implied unambiguous rankings.

Let us begin by assuming that there is a discount vector $\boldsymbol{X}=\left(x_{0}, x_{1}, \ldots, x_{n}\right)$, where $x_{i} \in$ $(0,1)$ denotes the discount factor applicable between period $i$ and period $i-1$, for $i=1, \ldots, n$, and $x_{0}$ is set equal to 1 . Let $\mathrm{D}:=\left\{\boldsymbol{X} \in \mathbb{R}^{n+1}: x_{0}=1\right.$ and $0<x_{i}<1$ for $\left.i=1, \ldots, n\right\}$ denote the set of all discount vectors. We can calculate overall discount factor, $y_{k}$, applicable to returns in period $i$ by multiplying the period-by-period discount rates as follows: $y_{k}=\prod_{i=0}^{k} x_{i}$ for $k=0,1, \ldots, n$. It is clear that $\boldsymbol{Y}:=\left(y_{0}, y_{1}, \ldots, y_{n}\right)$ is a strictly decreasing, strictly positive vector.

We are interested in finding necessary and sufficient conditions so that the present value of $\boldsymbol{a}$ is larger than the present value of $\boldsymbol{b}$ for all discount vectors $\boldsymbol{X}$. In symbols, we wish to find conditions on $\boldsymbol{c}=\boldsymbol{a}-\boldsymbol{b}$ such that

$$
\sum_{k=0}^{n} y_{k} c_{k}>0 \quad \text { for all } X \in \mathrm{D}
$$


Clearly this condition includes the previously studied case where $y_{k}=x^{k}$ for all $k$. Consequently, we know that necessary conditions (2) must apply, which require the first inequality $c_{0} \bullet 0$ and the last inequality $c_{0}+\ldots+c_{n} \geq 0$ of the partial sum condition ( $3 \mathrm{~b}$ ) to hold. It turns out that when discount rates can vary each period, condition (3b) is both necessary and sufficient for dominance.

THEOREM 7. Unambiguous dominance condition (5) holds if and only if

$$
\begin{aligned}
C_{k}:=\left(c_{0}+\ldots+c_{k}\right) \geq 0 \quad \text { for all } k=0,1, \ldots, n \\
\\
\text { with strict inequality for some } k .
\end{aligned}
$$

Proof. (Sufficiency) Suppose that the partial sum condition holds. Then by Abel's partial summation formula (Rudin, 1976, p. 70) we obtain

$$
\sum_{k=0}^{n} y_{k} c_{k}=C_{0}\left(y_{0}-y_{1}\right)+C_{1}\left(y_{1}-y_{2}\right)+\ldots .+C_{n-1}\left(y_{n-1}-y_{n}\right)+C_{n} y_{n}
$$

Since $\boldsymbol{Y}$ is strictly decreasing and strictly positive, all of the terms $\left(y_{0}-y_{1}\right), \ldots,\left(y_{n-1}-y_{n}\right)$ and $y_{n}$ are strictly positive. Therefore, dominance condition (5) holds.

(Necessity) Now, suppose that (5) is true. As before, $C_{0} \geq 0$ and $C_{n} \geq 0$ follow from the necessary conditions (2). Pick any $k^{\prime}=1, \ldots, n-1$, and consider any sequence $\boldsymbol{X}^{h} \in \mathrm{D}$ of discount vectors converging to $X^{0}$, where $x_{i}^{0}=1$ for $i \leq k^{\prime}$ and $x_{i}^{0}=0$ for $i \geq k^{\prime}+1$. Then by (5), we have $\sum_{k=0}^{n} y_{k}^{h} c_{k}>0$, so that in the limit $\sum_{k=0}^{n} y_{k}^{0} c_{k} \geq 0$. However, it is clear from the definition of $\boldsymbol{X}^{0}$ that $y_{i}^{0}=1$ for $i \leq k^{\prime}$ and $y_{i}^{0}=0$ for $i \geq k^{\prime}+1$, so that by Abel's formula, $\sum_{k=0}^{n} y_{i}^{0} c_{k}=C_{k^{\prime}} \geq 0$.

Consequently $C_{k} \geq 0$ for $k=0,1, \ldots, n$. Moreover, if we have $C_{k}=0$ for all $k$, then $c$ is the zero vector and dominance condition (5) clearly cannot hold. Therefore, strict inequality $C_{k}>0$ holds for some $k$. 
In our previous discussion of the case where the discount rate is fixed, condition ( $3 b)$ was found to be a sufficient, but not a necessary, condition for dominance. Theorem 7 shows that when we allow per-period discount rates to vary, the partial sum condition becomes necessary as well. Another way of viewing the result is that if dominance holds for all discount vectors, then it surely must hold for cases where the discount factor in a given period $k^{\prime}$ is close to zero. In these cases all subsequent period returns will have little weight in a calculation of present value. Consequently, it is as though we began with a net project with a horizon of $k^{\prime}$. And we know that the necessary conditions for a $k^{\prime}$ horizon project include the partial sum condition up to period $k^{\prime}$. Since this holds for all periods, we know that condition (3b) is necessary as well as sufficient.

Recall from our previous discussion that condition (3b) may be represented by the generalized concentration curves for projects $\boldsymbol{a}$ and $\boldsymbol{b}$, where the curve for $\boldsymbol{a}$ is somewhere above, and nowhere below, the curve of $\boldsymbol{b}$. Theorem 7 gives additional weight to the generalized concentration curve as a tool for evaluating projects: when the relationship between the curves holds, $\boldsymbol{a}$ must have a higher present value for any possible configuration of per-period discount factors.

The above approach rules out the possibility of equal preference across all periods or, indeed, a marked preference for future returns over current. The inequality $y_{i}>y_{i+1}$ is a key requirement of the above way of modeling a variable discount rate. As an alternative to constructing $y_{i}$ as a product of all previous per-period factors, we now simply posit $y_{i}>0$ to be the overall factor at which period $i$ returns will be discounted (for $i=0,1, \ldots, n)$. Let $\mathrm{D}^{\prime}:=\{\boldsymbol{Y} \in$ $\mathbb{R}^{n+1}: 0<y_{i}<1$ for $\left.i=1, \ldots, n\right\}$ denote the set of all vectors of overall discount factors $y_{i}$. The dominance condition then becomes

$$
\sum_{k=0}^{n} y_{k} c_{k}>0 \quad \text { for all } Y \in \mathrm{D}^{\prime}
$$


Condition (6) is clearly stronger than requirement (5), so a more restrictive condition on net project $c$ is likely to arise. Indeed, we now find that our most restrictive sufficient condition (3a) has become both necessary and sufficient.

THEOREM 8. Unambiguous dominance condition (6) holds if and only if

$$
\begin{aligned}
& c_{k} \geq 0 \quad \text { for all } k=0,1, \ldots, n \\
& \text { with strict inequality for some } k .
\end{aligned}
$$

Proof (Sufficiency) Suppose that $c_{k}$ are all non-negative and at least one is positive. Then since each $y_{k}$ is strictly positive, dominance condition (6) holds.

(Necessity) Now, suppose that (6) is true. Pick any $k^{\prime}=0, \ldots, n$, and consider any

sequence $\boldsymbol{Y}^{h} \in \mathrm{D}^{\prime}$ converging to $\boldsymbol{Y}^{0}$, where $y_{i}^{0}=1$ for $i=k^{\prime}$ and $y_{i}^{0}=0$ for $i \neq k^{\prime}$. Then by (6), we have $\sum_{k=0}^{n} y_{k}^{h} c_{k}>0$, so that in the limit $\sum_{k=0}^{n} y_{k}^{0} c_{k}=c_{k^{\prime}} \geq 0$. Consequently $c_{k} \geq 0$ for $k=0$, $1, \ldots, n$. Moreover, if $\boldsymbol{c}$ is the zero vector, then dominance condition (6) clearly cannot hold. Therefore, strict inequality $c_{k}>0$ holds for some $k$.

Theorem 8 reprises our most stringent sufficient condition and observes that it is also necessary for dominance when the weight on each period's returns is an arbitrary positive number less than one. This allows any single period to receive almost all the weight, which explains why nonnegative net returns in each period become necessary. The period-wise dominance of $\boldsymbol{a}$ over $\boldsymbol{b}$ is not particularly likely to hold. But when it does, Theorem 8 indicates the robustness of the dominance of $\boldsymbol{a}$ over $\boldsymbol{b}$ in present value terms.

\section{RELATED LITERATURE}

Our results have drawn from several strands of literature in mathematics, economics and finance. We now discuss this related work. 
The mathematical result underlying Theorem 5 has been known for some time. To the best of our knowledge, it made its first appearance in a note by Bernstein (1913), and was then restated in Bernstein (1915) in order to present a more precise form of a result of Laguerre. In 1952, this paper was translated from French into Russian and published as part of the collected works of Bernstein (see Academy of Sciences USSR, 1952). Hausdorff (1921, pp. 74-109) independently established that if a polynomial $P(x)=c_{0}+c_{1} x+\ldots+c_{n} x^{n}$ is positive on [0,1], then there exists an integer $m \geq n$ and positive coefficients $s_{0}, s_{1}, \ldots, s_{m}$ such that $P(x)$ can be represented as

$$
\sum_{k=0}^{m} s_{k} x^{k}(1-x)^{m-k}
$$

Note that this is an existence result, which says nothing about uniqueness and provides no definition of the coefficients $\left(s_{0}, \ldots, s_{m}\right)$ in terms of the underlying coefficients $\left(c_{0}, \ldots, c_{n}\right)$. Hardy, Littlewood and Polya (1952) in their classic book provided a generalization of Hausdorff's existence result, and referred to his 1921 paper, but not to Bernstein's earlier contribution. Hausdorff's result is also stated as problem 49 in G. Polya and G. Szego (1976, p. 78), and his solution is given on pp. 260-261.

Lorentz presented a systematic study of polynomials, which could be represented by (7), in a paper (1963) and subsequent book (1966). The representation, (7), of a polynomial, with $s_{k}$ unrestricted in sign, is referred to as a Lorentz representation of a polynomial by Milovanović , Mitrinovic and Rassias (1994). Erdelyi and Szabados (1988) refer to polynomials, which have a representation (7) with all $s_{k}$ non-negative or all $s_{k}$ non-positive, as Lorentz polynomials.

Cargo and Shisha (1966) state and prove Lemma 2, and call it (rightly) the Bernstein form of a polynomial. They attribute the result of Lemma 2 and its proof to Bernstein's paper, "On the best approximation of continuous functions by polynomials of a given degree", Communications of the Khar' kov Mathematical Society, Series 2, 13, (1912), 49-194.

Pratt (1979) provides a constructive proof to Lemma 2 that is almost identical to Bernstein's note in 1915. He refers to the non-constructive approach of Hausdorff (1921) and its 
generalization by Hardy, Littlewood and Polya (1952), but was apparently unaware of Bernstein's contributions to this subject. Pratt's results, dealing with the number of roots in $(0,1)$, are in fact more general than Bernstein's. He constructs an interesting tableau (similar to Pascal's triangle) as a practical device for detecting roots of a polynomial in $(0,1)$. A similar observation (although more restricted in scope than Pratt's tableau) also appears in Cargo and Shisha (1966). Erdelyi and Szabados (1988, Theorem 1), reprove representation result (7) with $s_{k} \geq 0$ for all $k$ as a corollary of their main approximation result. In a note added in proof, the authors state that they discovered, after preparing their manuscript, that this result is not new, and refer to Polya and Szego (1976, problem 49). Erdelyi (1991), provides refinements to the earlier approximation results of Erdelyi-Szabados, and attributes the representation result (7) with $s_{k} \geq$ 0 for all $k$ (this time correctly) to Bernstein's 1915 paper.

The possibility that one investment project might dominate another at all discount rates -the idea behind our dominance condition (1) -- has been part of the pedagogy surrounding the present value criterion for a long time. Recent examples of this include Mishan (1971) and Aronson (1985), both of whom provide numerical examples of projects that have this relationship. Fisher (1930) was also clearly aware of the possibility of dominance, and in fact took pains to convince his readers it was not the norm, i.e., that the ranking of alternatives according to present value generally depends on the rate of interest. Even though many authors were aware of the possibility of dominance, it appears that no one up to now has explored the extent to which dominance occurs, nor attempted to find a complete characterization of the induced partial ordering of projects.

Bøhren and Hansen (1980) and Ekern (1981) have studied the criterion of "time dominance" -- which uses an approach that is analogous to stochastic dominance in the analysis of risk (see, for example, Bawa (1975)). Recall that stochastic dominance unambiguously ranks lotteries in terms of higher expected utility for all utility functions in a given class (usually defined by restrictions on the derivatives of utility). Time dominance unambiguously ranks projects in terms of higher present value for all discounting functions (of time) drawn from a 
particular class (defined by restrictions on the derivatives of the discounting function with respect to time). The resulting rankings are then characterized using techniques analogous to those employed in the stochastic dominance literature. In particular, Bøhren and Hansen (1980, Proposition 1) show that when the discounting function has a negative first derivative and hence exhibits a positive time preference, one obtains a result analogous to Theorem 7 above. They also note (in Proposition 3) that since each of the classes includes the discounting functions associated with fixed discount rates, each is a sub-relation of the partial ordering defined in (1). However, the characterization of unambiguous ranking (1) is not the aim of the "time dominance" literature.

There are clear links between our results and the literature on internal rates of return. Fisher (1930) introduced the notion of the "rate of return over cost" as the rate of discount at which two projects have the same present value or, equivalently, the rate at which the net project has zero present value (hence the net project's internal rate of return). This rate was then used as a cutoff between the range of discount rates that select one project and the range of rates that select the other. Our dominance criterion (1) is thus equivalent to requiring that there is no positive rate of return over cost. ${ }^{12}$

As emphasized by both Fisher (1930) and Alchian (1955), the internal rates of return of the individual projects themselves are not particularly relevant to project selection. Alchian in particular provides a simple example in which one project has a higher internal rate of return than another, and yet there is a wide range of discount rates at which the opposite ranking holds in terms of present value (namely, all those lower than the rate of return over cost). In fact, it is not difficult to construct examples for which one project dominates a second project at all discount rates and yet the second has a higher internal rate of return. ${ }^{13}$ This provides even more

12 Our criterion is also related to the "reswitching" debate in capital theory (see, for example, Bruno, Burmeister and Sheshinski (1966)), wherein projects switch orders twice as the prevailing rate changes. Dominance rules out any re-ranking of the two projects as the interest rate changes.

${ }^{13}$ Consider $\boldsymbol{a}=(9,4,-17)$ and $\boldsymbol{b}=(5,8,-17)$. It can be verified that the present value of $\boldsymbol{a}$ exceeds the present value of $\boldsymbol{b}$ for all discount factors $x \in(0,1)$. However, the internal rate of return of $\boldsymbol{a}$ 
reason for present value maximizers to ignore a project's internal rate of return, if indeed one exists.

Pratt and Hammond (1979) construct a procedure for finding an upper bound on the number of internal rates of return for a given project. They consider differences in projects and, like Fisher (1930), concentrate on the resulting "break-even" rate at which the present values of the two projects are the same. They note that when no break-even rate exists, "one project dominates the other, being preferable at all interest rates," and offer sufficient conditions (3b) as partial characterization of this dominance (Rule 3a, p.1234). Their general procedure is analogous to that used in Pratt (1979) and hence is closely related to Bernstein (1915) and Lemma 2 above.

\section{CONCLUDING REMARKS}

In this paper we have proposed a criterion that ranks projects independent of the discount rate. The criterion leads to a partial ordering of projects that is intuitive and reasonably applicable. We presented several characterizations of the basic ranking, including complete characterizations based on the approach of Bernstein (1915). We also showed how the ranking is altered when the overall range of discount rates is restricted or the rate varies period by period.

One important lesson to be drawn from this paper is that unambiguous dominance is by no means impossible, and that limited range comparisons are more likely still. Consequently, we would argue that any project evaluation based on a specific discount rate should include information on whether, and to what extent, the initial judgment is robust to changes in the

is $17 \%$, while the internal rate of return of $\boldsymbol{b}$ is $21 \%$. The key characteristic of these projects is that, in the words of Fisher (1930), that "the advantages (returns) precede the disadvantages (costs)." See also Bierman and Smidt (1980) who call such projects "loan-type" investments. For such projects, there has been some discussion in the literature about whether they can be truncated at a date prior to the terminal date to avoid the negative returns. Sen (1975) has argued, using the application of DDT as an example, that the "disadvantage" (pollution) is causally linked to the use of DDT, and (costless) truncation of the project, after the "advantage" (increased crop production) has been reaped, may not make such sense. 
discount rate. If unambiguous dominance holds, then for purposes of comparing projects, the discussion is ended. If there is dominance for a range of rates, then the discussion should center on whether the range includes all "reasonable" rates. In any case, it would not be difficult to write a program to check whether the above conditions hold. Alternatively, one could obtain an approximate answer to the question of robustness by simply plotting the present value polynomial on the unit interval for a reasonably fine grid level. Irrespective of how the approach is eventually implemented, we feel that it could be an important part of the standard toolkit for evaluating projects. ${ }^{14}$

We would also like to mention a number of potential directions for future work. One possible criticism of the conditions underlying Theorem 5 is that even if one project unambiguously dominates another, there is no assurance that the condition would be able to detect this fact for $m$ reasonably small. For example, the net project $c=(1,-6,10,1)$ satisfies criterion (1) for unambiguous dominance, but it takes $m \geq 18$ to detect this fact using the test from Theorem 5. It turns out that the polynomial generated by $c$ brushes very close to 0 , causing the approximating function $f^{m}$ to dip below the axis for the initial levels of $m$. While the likelihood of this occurring in practice may be low, it would still be an interesting theoretical question to see whether $m$ of Theorem 5 might be derivable as well. ${ }^{15}$

We have assumed throughout that time is discrete and finite, which has allowed us to make use of the theory of polynomials in our characterization results. It would be interesting to see what form necessary and sufficient conditions would take if time were modeled as a continuous or an unbounded variable. The extension to uncertain cash flows would likewise be valuable, as would the case where the discount rate (or the range of possible discount rates) varies over time. Finally, in defining the dominance criterion we intentionally ignored the feasibility of the projects under consideration. Yet we can imagine that considerations of this

${ }^{14}$ This is precisely what happened to the variable poverty line "poverty orderings" developed by Foster and Shorrocks (1988a, 1988b).

${ }^{15}$ In fact, there is a body of literature in mathematics -- on the "Lorentz number" -- that may be directly applicable to this question. 
type might actually broaden the possibility for comparisons. For suppose that two projects are deemed non-comparable, in that one dominates the other for one range of discount rates with the opposite occurring over another range. Consider a case in which the only discount rates for which the second project dominated the first occurred where both projects had a negative present value. Then, there might be grounds for concluding that the first dominates the second. It would be interesting to characterize the resulting hybrid partial ordering. 


\section{APPENDIX}

Proof of Lemma 2. Pick any $\varepsilon>0$. Select $\alpha \in \mathbb{R}$ satisfying $\alpha>\max \left\{\left|c_{0}\right|,\left|c_{1}\right|, \ldots,\left|c_{n}\right|, \varepsilon\right\}$ and define $\beta:=\varepsilon /(2 n \alpha)$. Note that since $n \geq 1$ and $0<\varepsilon<\alpha$, we have $0<\beta<1 / 2$, and hence 0 $<1-(1-2 \beta)^{1 / 2}<1$. Now define $m(\varepsilon)$ so that

$$
m(\varepsilon)>\left[1-(1-2 \beta)^{1 / n}\right]^{-1} n / \beta
$$

and let $m$ be any integer satisfying $m \geq m(\varepsilon)$. We will now show that

$$
\left|\left[s_{k}^{m} /\left(\begin{array}{c}
m \\
m-k
\end{array}\right)\right]-P_{c}\left(\frac{k}{m}\right)\right|<\varepsilon \quad \text { for all } k=0,1, \ldots, m .
$$

Consider any $k=0,1, \ldots, m$. By definition,

$$
P_{c}\left(\frac{k}{m}\right)=c_{0}+\left(\frac{k}{m}\right) c_{1}+\ldots+\left(\frac{k}{m}\right)^{n} c_{n}
$$

and

$$
\left[s_{k}^{m} /\left(\begin{array}{c}
m \\
m-k
\end{array}\right)\right]=c_{0}+\left[\left(\begin{array}{c}
m-1 \\
m-k
\end{array}\right) /\left(\begin{array}{c}
m \\
m-k
\end{array}\right)\right] c_{1}+\ldots+\left[\left(\begin{array}{c}
m-k^{\prime} \\
m-k
\end{array}\right) /\left(\begin{array}{c}
m \\
m-k
\end{array}\right)\right] c_{k^{\prime}}
$$

where $k^{\prime}=\min \{k, n\}$. Note that for $j=1, \ldots, n$, we have

$$
\left[\left(\begin{array}{c}
m-j \\
m-k
\end{array}\right) /\left(\begin{array}{c}
m \\
m-k
\end{array}\right)\right]=\frac{(m-j) ! k !}{(k-j) ! m !}=\frac{k(k-1) \cdots(k-j+1)}{m(m-1) \cdots(m-j+1)} .
$$

We divide our analysis into two cases. First, suppose that $k<\beta m$. Note that $\left|c_{j}\right|$ $\leq \alpha,\left(\begin{array}{c}m-j \\ m-k\end{array}\right) /\left(\begin{array}{c}m \\ m-k\end{array}\right) \leq \frac{k}{m}$ and $\left(\frac{k}{m}\right)^{j} \leq \frac{k}{m}$ for $j=1, \ldots, n$. Thus,

$$
\begin{aligned}
\left|\left[s_{k}^{m} /\left(\begin{array}{c}
m \\
m-k
\end{array}\right)\right]-P_{c}\left(\frac{k}{m}\right)\right| & \leq\left|\left[s_{k}^{m} /\left(\begin{array}{c}
m \\
m-k
\end{array}\right)\right]-c_{0}\right|+\left|P_{\boldsymbol{c}}\left(\frac{k}{m}\right)-c_{0}\right| \\
& \leq \sum_{j=1}^{k^{\prime}}(k / m)\left|c_{j}\right|+\sum_{j=1}^{n}(k / m)\left|c_{j}\right| \\
& \leq\left(k^{\prime}+n\right)(k / m) \alpha \leq 2 n \beta \alpha=\varepsilon,
\end{aligned}
$$

where use has been made of the restriction on $k$.

Alternatively, suppose that $k \geq \beta m$. Then according to (A1) we have $k \geq \beta m(\varepsilon)$ $>n$ and consequently $k^{\prime}=n$. It follows that

$$
\begin{aligned}
\left|\left[s_{k}^{m} /\left(\begin{array}{c}
m \\
m-k
\end{array}\right)\right]-P_{\boldsymbol{c}}\left(\frac{k}{m}\right)\right| & \leq \alpha \sum_{j=1}^{n}\left|\left[\left(\begin{array}{c}
m-j \\
m-k
\end{array}\right) /\left(\begin{array}{c}
m \\
m-k
\end{array}\right)\right]-\left(\frac{k}{m}\right)^{j}\right| \\
& =\alpha \sum_{j=1}^{n}(k / m)^{j}\left|\prod_{i=0}^{j-1} \frac{(1-i / k)}{(1-i / m)}-1\right| .
\end{aligned}
$$


However, for $j=1, \ldots, n$, we have

$$
\begin{aligned}
1 \geq \prod_{i=0}^{j-1} \frac{(1-i / k)}{(1-i / m)} & \geq \prod_{i=0}^{j-1}(1-i / k) \geq(1-n / k)^{n} \geq[1-(n /(\beta m(\varepsilon)))]^{n} \\
> & 1-2 \beta
\end{aligned}
$$

where use has been made of (A1). Therefore,

$$
\left|\left[s_{k}^{m} /(\underset{m-k}{m})\right]-P_{c}\left(\frac{k}{m}\right)\right| \leq \alpha n 2 \beta=\varepsilon,
$$

which completes the proof. 


\section{REFERENCES}

J. R. Aronson, "Public Finance," McGraw-Hill, New York, 1985.

Academy of Sciences USSR, "Collected Papers of S.N. Bernstein, vol. 1, Constructive Theory of Functions (1905 - 1930)," Moscow, 1952.

A. Alchian, The rate of interest, Fisher's rate of return over costs and Keynes' internal rate of return, American Econ. Rev. 45 (1955), 938-943.

V. S. Bawa, Optimal rules for ordering uncertain prospects, J. Financial Econ. 2 (1975), 95-121.

O. Bøhren and T. Hansen, Capital budgeting with unspecified discount rates, Scand. J. of Econ. 82 (1980), 45-58.

S. Bernstein , Sur les séries normales, in "Lecons sur Les Principes de L'Analyse, Volume 2," by R. d'Adhémar, Gauthier-Villars, Paris, 1913.

S. Bernstein, Sur la representation des polynomes positifs, Soobsc. Har'k Matem. Ob., Ser. 214 (1915), 227-228, reprinted (and translated into Russian) in Academy of Sciences USSR (1952), 251-252.

H. Bierman, Jr., and S. Smidt, “The Capital Budgeting Decision,” fifth edition, MacMillan, New York, 1980.

M. Bruno, E. Burmeister and E. Sheshinski, The Nature and Implications of the Reswitching of Techniques, Quarterly Journal of Economics, 80 (1966), 526-553.

G. T. Cargo and O. Shisha, The Bernstein form of a polynomial, J. of Research of the National Bureau of Standards - B. Mathematics and Mathematical Physics, 70 B (1966), 79-81.

L. E. Dickson, "New First Course in the Theory of Equations," Wiley, New York, 1939.

S. Ekern, Time dominance efficiency analysis, J. of Finance 36 (1981), 1023-1034.

T. Erdelyi, Estimates for the Lorentz degree of polynomials, J. of Approximation Theory 67 (1991), 187-198.

T. Erdelyi and J. Szabados, On polynomials with positive coefficients, J. of Approximation Theory 54 (1988) 107-122.

I. Fisher, "The Theory of Interest," Macmillan, New York, 1930.

J. E. Foster and A. F. Shorrocks, Poverty orderings, Econometrica 56 (1988a), 173-77.

J. E. Foster and A. F. Shorrocks, Poverty orderings and welfare dominance, in "Distributive Justice and Inequality: A Selection of Papers Given at a Conference, Berlin, May 1986," (W. Gaertner and P.K. Pattanaik, Eds.), Springer, New York, 1988 b.

G. H. Hardy, J. E. Littlewood, and G. Polya, "Inequalities," Cambridge University Press, New York, 1952. 
F. Hausdorff, Summationsmethoden und momentfolgen, I, Mathematische Zeitschrift 9 (1921) 74-109.

J. Hirshleifer, On the Theory of Optimal Investment Decision, The Journal of Political Economy, 66 (1958), 329-352.

P. J. Lambert, "The Distribution and Redistribution of Income: A Mathematical Analysis," 2nd edition, Manchester University Press, Manchester, 1993.

K. Knopp, "Problem Book in the Theory of Functions," Dover, New York, 1952.

R. C. Lind, "Discounting for Time and Risk in Energy Policy," Resources for the Future Publication; The Johns Hopkins Press, Baltimore, Maryland, 1982.

G. G. Lorentz, The degree of approximation of polynomials with positive coefficients, Math. Ann. 151 (1963), 239-251.

G. G. Lorentz, "Approximation of Functions," Holt, Rinehart and Winston, New York, 1966.

G. V. Milovanovic , D.S. Mitrinović and Th. M. Rassias, "Topics in Polynomials: Extremal Problems, Inequalities, Zeros," World Scientific, Singapore, 1994.

E. J. Mishan, "Cost-Benefit Analysis," Praeger, New York, 1971.

J. W. Pratt, Finding how many roots a polynomial has in $(0,1)$ or $(0, \bullet)$, American Mathematical Monthly 86 (1979) 630-637.

J. W. Pratt and J.S. Hammond, III, Evaluating and comparing projects: simple detection of false alarms, J. of Finance 34 (1979), 1231-1242.

G. Polya and G. Szego, "Problems and Theorems in Analysis, Volume II," Springer-Verlag, Berlin, 1976.

W. Rudin, "Principles of Mathematical Analysis," third edition, McGraw-Hill, New York, 1976.

A. Sen, Minimal Conditions for Monotonicity of Capital Value, Journal of Economic Theory, 11 (1975), 340-355.

A. Sen, "On Economic Inequality," enlarged edition, Clarendon Press, Oxford, 1997.

A. F. Shorrocks, Ranking income distributions, Economica 50 (1983), 3-17.

J. V. Uspensky, "Theory of Equations," McGraw-Hill, New York, 1948. 


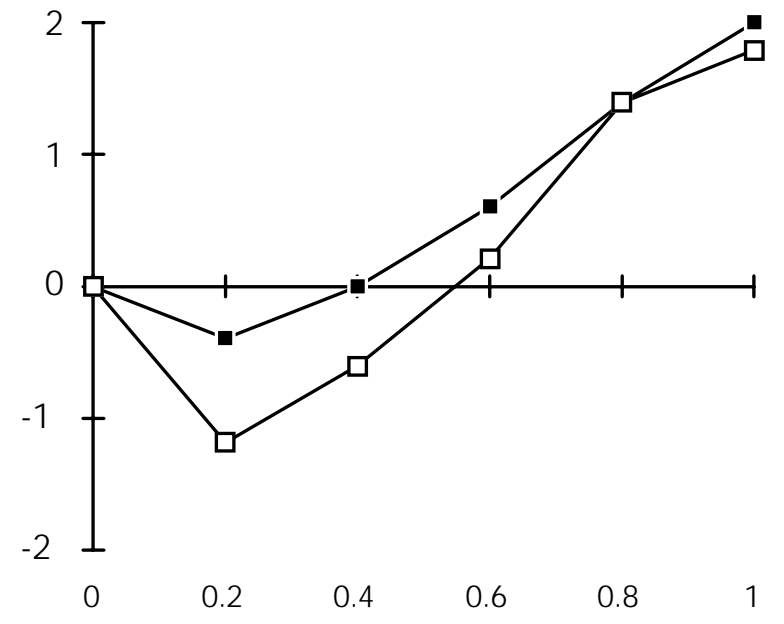

FIG. 1. Generalized concentration curves. 


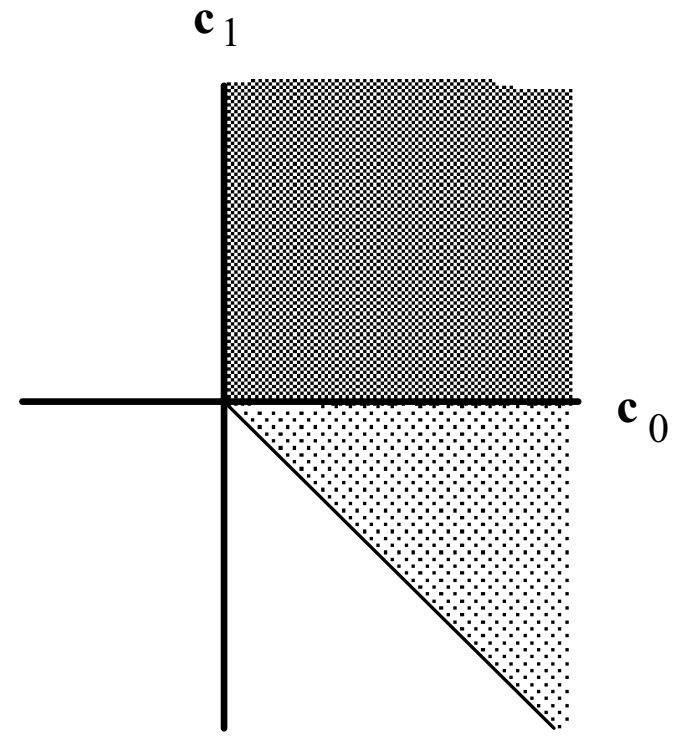

FIG. 2. Dominance for $\mathrm{n}=1$. 


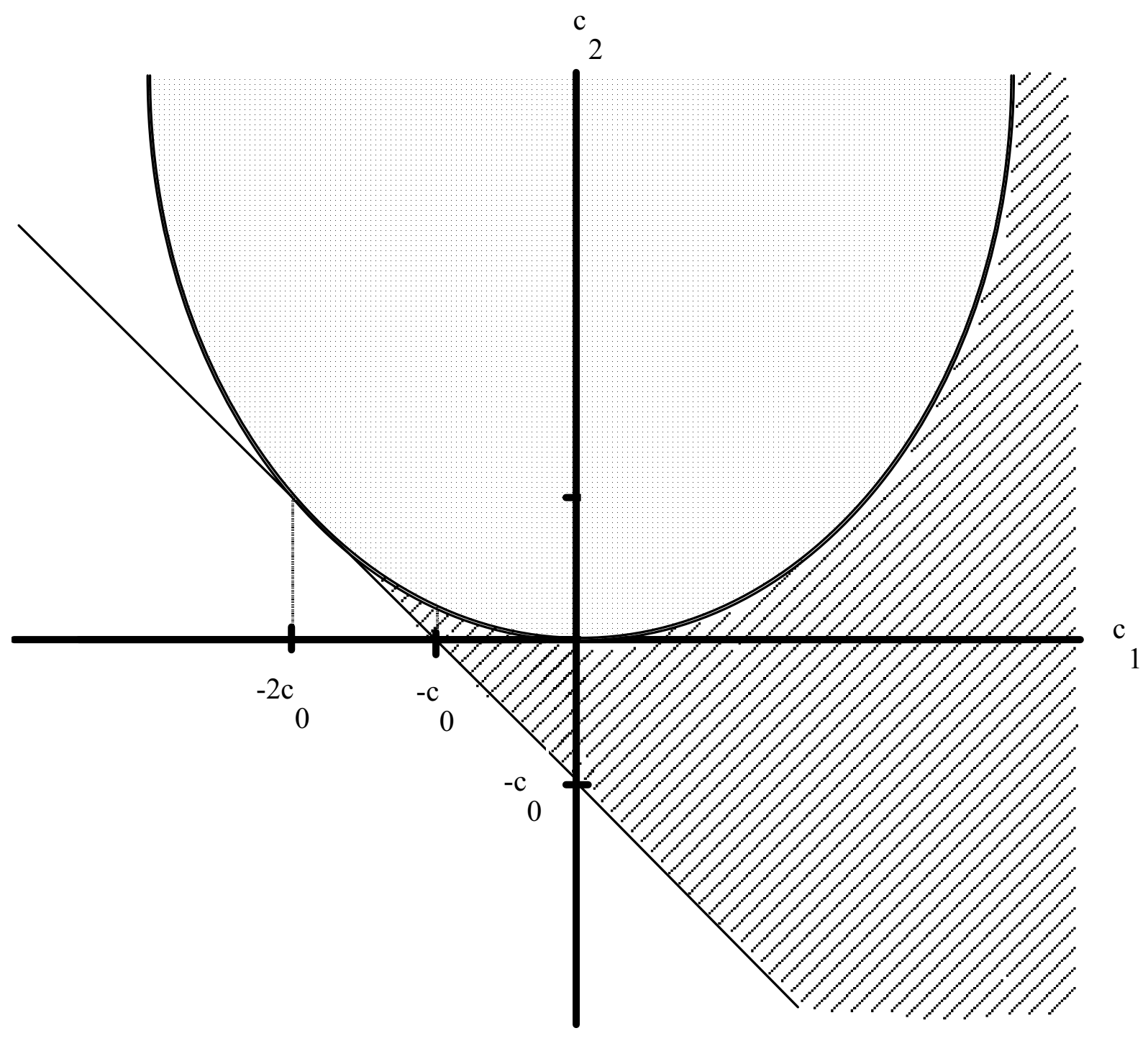

FIG. 3. Dominance for $n=2$. 


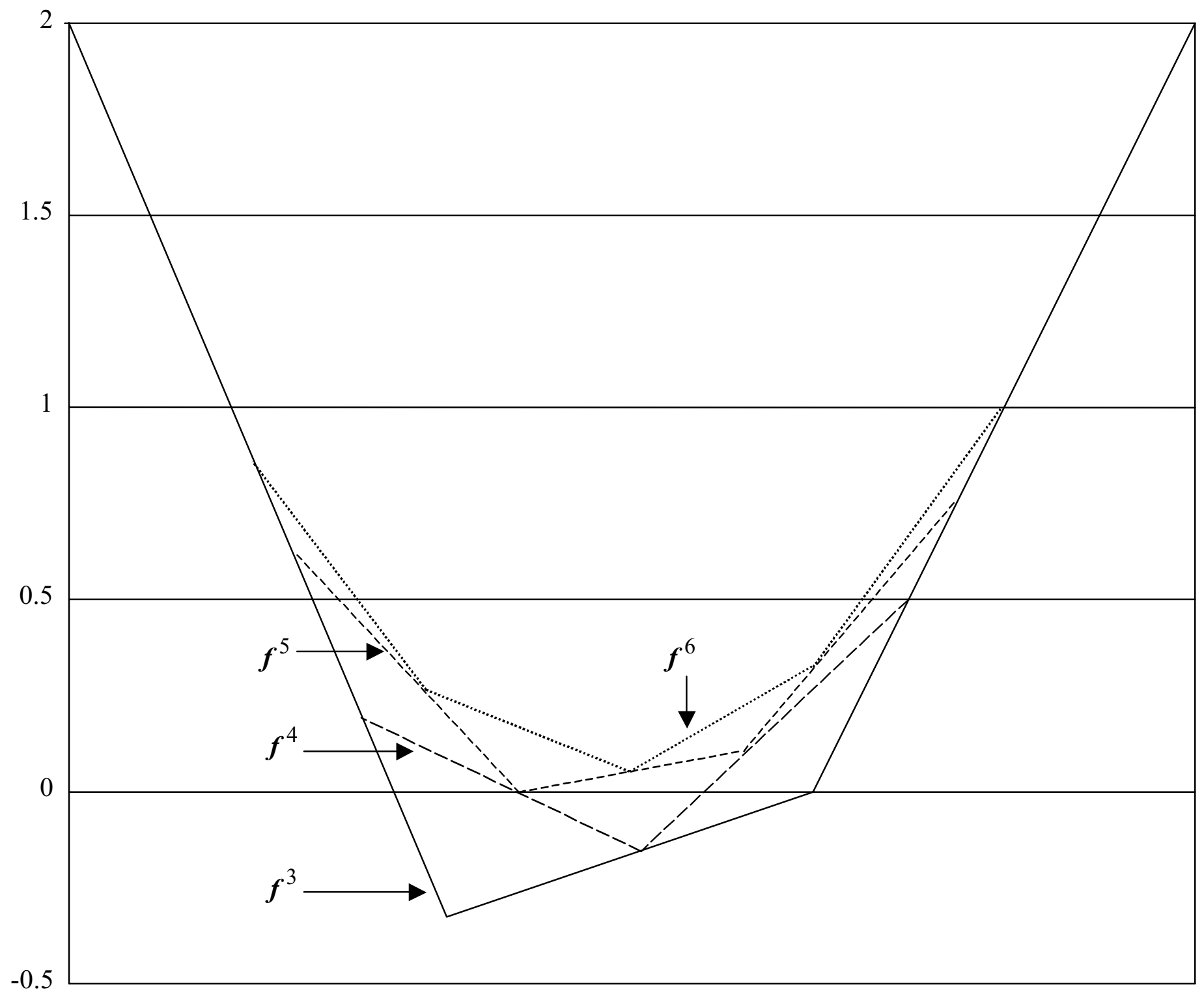

FIG. 4. Successive approximations and dominance. 